
the conditions of the Creative Commons Attribution-NonCommercial-ShareAlike licence $<$ http://creativecommons.org/licenses/by-nc-sa/3.o/ > . The written permission of Cambridge University Press must be obtained for commercial re-use.

doi:10.1017/So144686X12001511

\title{
A support network typology for application in older populations with a preponderance of multigenerational households
}

\author{
VANESSA BURHOLT* and CHRISTINE DOBBS*
}

\begin{abstract}
This paper considers the support networks of older people in populations with a preponderance of multigenerational households and examines the most vulnerable network types in terms of loneliness and isolation. Current common typologies of support networks may not be sensitive to differences within and between different cultures. This paper uses cross-sectional data drawn from 590 elders (Gujaratis, Punjabis and Sylhetis) living in the United Kingdom and South Asia. Six variables were used in K-means cluster analysis to establish a new network typology. Two logistic regression models using loneliness and isolation as dependent variables assessed the contribution of the new network type to wellbeing. Four support networks were identified: 'Multigenerational Households: Older Integrated Networks', 'Multigenerational Households: Younger Family Networks', 'Family and Friends Integrated Networks' and 'Non-kin Restricted Networks'. Older South Asians with 'Non-kin Restricted Networks' were more likely to be lonely and isolated compared to others. Using network typologies developed with individualistically oriented cultures, distributions are skewed towards more robust network types and could underestimate the support needs of older people from familistic cultures, who may be isolated and lonely and with limited informal sources of help. The new typology identifies different network types within multigenerational households, identifies a greater proportion of older people with vulnerable networks and could positively contribute to service planning.
\end{abstract}

KEY WORDS-social resources, support networks, ethnic minority groups, immigration, loneliness, social isolation, BME, multigenerational households.

* Centre for Innovative Ageing, College of Human and Health Sciences, Swansea University, UK. 


\section{Introduction}

Support networks are made up of different configurations of relationships and represent the matrix of sources of social, emotional and instrumental support and help available to an older person. However, it is doubtful as to whether the common typologies of support networks are 'fit for purpose' in all of the studies in which they are utilised. Lubben and Gironda (2003) have noted that the instruments that assess social support should be considered in relation to their sensitivity to differences within and between different cultures. Whereas previously many societies could be considered culturally homogenous, now many communities are culturally heterogeneous and comprise people from other countries or communities, with different backgrounds, religion and race. In this respect we should challenge some of the typologies that have been developed for 'majority' or indigenous populations in Western societies, and that have not been extensively tested for use with ethnic minority groups nor with those people living in other non-Western countries (Lubben and Gironda 2003). However, in the gerontological literature there has been little attention paid to the suitability of network typologies for use in other cultures, particularly in those where multigenerational living arrangements are common. This should be of concern to gerontologists and policy makers working with these populations.

\section{Studies of support networks}

Specific instruments to assess social support networks have been developed for use with older populations. Some of the most commonly used include the Wenger Support Network Typology (Wenger 1991), The Lubben Support Network Scale (LSNS; Lubben and Gironda 2004; Lubben et al. 2006) and Litwin Support Network Types (Litwin $1997 a)$. Whereas the LSNS computes a scale to gauge social isolation and levels of perceived social support from family and friends, Wenger's Support Network Typology and Litwin's Support Network Types categorise social support relationships into five groups based on the availability of local kin, frequency of face-to-face interaction with family, friends and neighbours, and integration in community and religious groups (Litwin 1997a; Wenger 1991). While the LSNS and Wenger Support Network Typology can be used by practitioners in the field (as they comprise questions that can be scored), the Litwin typology requires cluster analysis to identify network types. Despite differences, there are common threads within each, namely the frequency of contact with, or proximity of children. 


\section{Vanessa Burholt and Christine Dobbs}

To date, using existing support network typologies to study the social resources of some groups of older people has been problematic. The Wenger typology has been used to identify the support network types of older South Asian and South-East Asian elders in their countries of origin (Bangladesh: Burholt et al. 2000, 2003; China: Wenger and Liu 1999; India: Burholt et al. 2003; Nepal: Shrestha 2006) and as migrant populations living in the United Kingdom (UK) (Bangladeshis: Burholt et al. 2000, 2003; Indians: Burholt et al. 2003). In this research more than three-quarters of all respondents (indigenous or migrant populations) were categorised as having either family-dependent or locally integrated support networks. In Western populations, these two networks provide the highest levels of informal care. However, the classification in Asian populations is likely to have been unduly influenced by the proximity and frequency of contact of family members within multigenerational households. We do not know if South Asian families within multigenerational households also provide support.

The proximity and/or frequency of contact with children are essential components in the commonly employed typologies of social support for older people (e.g. Litwin 1997a; Lubben and Gironda 2004). These variables have been useful in delineating network types within cultures that predominantly comprise nuclear households or single-person households and where co-residence (of three or more generations) is uncommon. However, it is clear that while nuclear or single-person households may be common for older people in Northern Western Europe (Tomassini et al. 2004), the United States of America (Tomassini et al. 2004) and Australia (Paice 2002), they are not representative of living arrangements in developing countries (Ruggles and Heggeness 2008), Asia (e.g. Burholt et al. 2003; Sereny 2011), Eastern, Southern or Central Europe (Tomassini et al. 2004; Wilmoth 2001), Central or South America (Wilmoth 2001), or for migrants from these countries (Wilmoth 2001).

In other cultures where multigenerational households are common, caring for parents has been discussed often in terms of filial obligation which is described as a sense of duty towards one's parents. It has been argued that in countries that emphasise interdependence or filial obligation, merely 'being old' is sufficient for a younger generation to provide help, whereas in countries that emphasise independence as a goal, help is only forthcoming in the face of need (e.g. ill health) (Liu and Kendig 2000). Current classifications of support network types originating from individualistically oriented Western countries may not adequately capture the ranges of experiences of older people from cultures with familistic traditions. We cannot assume that intergenerational co-residence equates to the provision of support for older people: older people may be providing support undertaking 
household tasks for others (Lowenstein 2002), or providing care to younger generations (Minkler 1999), or they may have a lower priority given to their support needs than others in the household ( Ng, Phillips and Lee 2002; Treas and Mazumdar 2002).

There have been few published attempts to classify the network types of older migrants (e.g. Russian Jews: Litwin 1995, 1997b) or those living in non-Western communities (e.g. in Mexico: Dubova et al. 2010; in China: Cheng et al. 2009) with familistic values. Where new typologies have been developed the authors have not considered why the classification of networks of older people from these cultures should differ from individualistically oriented countries (except Cheng et al. 2009), or how the network classifications may be used in a wider context (e.g. in other cultures with similar normative household forms).

As the operationalisation of network types in familistic cultures has been limited, theoretical approaches to the study of social support networks of older people living in multigenerational households are also somewhat restricted. Modernisation theory (Cowgill 1986) has been applied to the study of social support in developing countries and for emigrants from these countries, while minority group theory (Wirth 1945) has focused on marginalisation from mainstream society for older migrants, and the impact that this may have on care and support.

Modernisation theory suggests that particular family forms are associated with the stage of economic development and urbanisation of a country. Thus, developing countries are assumed to have familistic values where elders are held in high regard and a greater proportion of the population live in 'traditional' multigenerational households (Yeo and GallagherThompson 2006). The transition from traditional to modern society is accompanied by rapid increases in knowledge and high levels of occupational specialisation. Migration in search of appropriate education and employment is assumed to result in greater geographic separation between generations within families and a decline in contact between older parents and their adult children (Silverstein et al. 1998). Thus, according to modernisation theory, modern societies are more likely to have individualistically oriented cultures that encourage independence, provide lower levels of support and be characterised by nuclear households where co-residency with older parents is uncommon (Rosenthal 1986).

Minority group theory suggests that particular traits such as race are used to marginalise certain groups from society (Wirth 1945). Gerontologists have used minority group theory to explain ageism (Palmore 1978) and have suggested that characteristics or traits such as wrinkles and grey hair that mark an individual as 'old' can result in the application of other agerelated stereotypes including negative evaluations of competence (Kite et al. 


\section{Vanessa Burholt and Christine Dobbs}

2005). This in turn can lead to ageism, discrimination and marginalisation (Levin and Levin 1982). In addition to the risk for ageism, older people from ethnic groups may have been excluded from educational or high-status occupational opportunities across the lifecourse because of their racial identity (Hendricks and Hendricks 1977; Palmore and Manton 1973). Inequalities in opportunities can negatively impact on health and economic resources in later life, and in turn impact on the ability to provide care to others (Willis 2008). Moreover, multigenerational households are another characteristic distinct from normative residency patterns that may be used to marginalise groups, whereby co-residency and assumed levels of social support are provided as a justification for reduced access to appropriate formal care services (Willis 2010).

There is little empirical evidence to refute or support the tenets of modernisation theory or minority group theory in relation to the supposed impacts on support networks. While inductive qualitative studies are extremely useful to provide an understanding of the experience of care and social support in multigenerational households, robust instrumentation is required so that positivistic and deductive approaches can also be adopted to test hypotheses and challenge existing theoretical perspectives.

\section{Support networks and migration}

It is commonly assumed that migration impacts on the social and support networks of migrants, inasmuch as support networks need to be reconstituted following relocation (Rogler 1994). However, each ethnic group is 'a collectivity within a larger society having a real or common ancestry, memories of a shared historical past, and a cultural focus' (Schermerhorn 1970: 12). In this respect, shared beliefs, norms, values and preferences for living arrangements and support in later life are likely to persevere beyond the boundaries of the country of origin and have an enduring impact on the lives of migrants. We would expect 'ethnic heritage' to influence participation and the nature of informal care systems for South Asian migrants in the UK (Thornton and White-Means 2000). Simultaneously, we would expect the mainstream culture and infrastructure in the UK (such as care-giving norms, gender equality, and the health and welfare system) to influence migrants' behaviours and alter family structure and function especially with regard to the availability of informal support for older people (Burholt and Dobbs 2010; Holmes and Holmes 1995).

\section{Support networks and wellbeing}

Social support is important to people at all stages of the lifecycle and its contribution to wellbeing has been well documented (Cheng et al. 2011). 
However, there is no consensus on what constitutes wellbeing for older people, or how it should be measured. Conceptualisations of wellbeing vary between scientific disciplines, but within sociology and psychology it is broadly defined as being in a 'good state' (Veenhoven 2004). In the study of older people, wellbeing is commonly operationalised as psychological, physical or material wellbeing (e.g. Paim 1995; Ryff and Keyes 1995).

In the human sciences, several concepts are used synonymously with wellbeing such as happiness (Blanchflower and Oswald 2004), morale (Lawton 1975), quality of life (Slocum-Gori et al. 20og), life satisfaction (Diener, Diener and Diener 1995) and positive affect (Lawton 1983). Objective measures such as functional health are also used imprecisely to capture wellbeing (George 2010). Some authors suggest that wellbeing is multidimensional (Ryff and Keyes 1995; Slocum-Gori et al. 2009) and, for example, can be expressed in relation to the perceived quality of relationships with others and the immediate community or environment (Keyes 1998). Whereas, in general, multidimensional measures capture concepts more precisely than single indicators, this is not the case for wellbeing as single items are also valid and reliable (George 2010).

In a review of the literature on wellbeing in sociological and psychological journals, George (2010) identified five theoretical approaches in gerontological research: discrepancy theories; social comparison theory; strategic investment of resources (such as selective optimisation theory; Baltes and Carstensen 2003); social stratification of wellbeing; and a social indicators perspective. This paper draws on two theoretical approaches - discrepancy theory and social stratification - to capture wellbeing. Although there are context-free subjective and objective composite instruments that assess wellbeing, the current analysis focuses on two single-item wellbeing outcomes: loneliness and isolation (see also Litwin and Shiovitz-Ezra 2011 ; Lubben and Gironda 2000, 2003). These are context-specific measures of wellbeing focusing on a single area of life concerned with social relationships (Blanchflower and Oswald 2004).

Discrepancy theories suggest that positive feelings of wellbeing result when there is little difference between aspirations and achievements. Conversely, negative wellbeing results from significantly under-achieving in relation to one's aspirations (George 2010). In this paper, loneliness is defined as a subjective state of mind concerning negative feelings about the discrepancy between the desired and achieved level of social contact (Wenger and Burholt 2004). Thus, loneliness can be considered as an element of the social dimension of wellbeing from a discrepancy perspective.

Social stratification of wellbeing suggests that wellbeing is related to resources, with those allocated greater resources having more positive experiences than those with fewer resources. In this respect, in this paper social 


\section{Vanessa Burholt and Christine Dobbs}

isolation is a more objective concept than loneliness that we describe as the absence of contact with other people at one extreme versus high levels of social contact at the other extreme (Wenger and Burholt 2004). With regard to social stratification theory, it could be argued that those with greater levels of social resources occupy a more advantaged position and will have the highest levels of wellbeing. Whilst we acknowledge that a variety of factors impact on wellbeing, there is a body of evidence that suggests that robust support networks act as a buffer against the effects of loneliness and isolation (Cattell 2001; Golden, Conroy and Lawlor 2009; Lubben and Gironda 2003; Wenger 1997). Conversely, weaker support networks are more prone to adverse wellbeing outcomes and as such have been targeted in the delivery of personal, social and health-care services (Wenger and Tucker 2002).

\section{Research questions}

The composition of the most commonly used support network measures cited above suggests that multigenerational living arrangements could impact on the classification of network types because of the proximity of, and frequency of, contact with relatives living in the same house. A skew towards more robust networks in the classification and distribution of network types may result in the overestimation of the levels of wellbeing, and an underestimation of the need for formal service provision in populations where intergenerational co-residence is common (e.g. Cooper, Bebbington and Livingston 2010). Given the deficit of knowledge regarding the suitability of current network typologies for use in familistic cultures, this paper seeks to answer one main research question: can a new robust network typology be discerned in older populations with a preponderance of multigenerational or extended households? We supplement this primary question with a secondary question: can a new network typology be validated through its predictive utility? To address the latter question, we examine the associations between the derived typology, migrant status and the Wenger Support Network Typology to see if meaningful differences are found. Furthermore, we look at the ability of the network typology to predict outcomes that are unrelated to the variables used in the clustering, but that are theoretically related to the clusters (Henry, Tolan and Gorman-Smith 2005): we determine whether network type predicts loneliness and isolation.

\section{Methods}

Sample

The analysis is based on data from the 'Families and Migration: Older People from South Asia' project (for further details of the project, see Burholt, 
2004a, 2004 $b$; Burholt and Dobbs 2010; Burholt and Shah 2001; Burholt and Wenger 2004). The sample included 303 migrant elders (age 55 years or more) in the UK (103 Gujaratis, 100 Punjabis and 100 Sylhetis) and 287 older people in Asia (10o each of Gujaratis and Indian Punjabis and 87 Sylhetis). The total sample of 590 was stratified $5^{0}: 5^{\circ}$ by gender. This dataset is unique in several respects: (a) it is one of the largest datasets of older migrants in the UK; (b) it comprises comparable data on older South Asians for those that have migrated from and those that have remained living in South Asia; and (c) it contains demographic data for each of $\mathrm{N}=4,648$ network members.

Beauchemin and González-Ferrer (2011) note that there are several problems associated with obtaining a representative sample of migrants. Migrants are usually a relatively rare population, they may be undocumented and difficult to find and, therefore, sample frames are rarely available. Consequently, snowballing or chain-referral methods of building a sample are the preferred techniques adopted by researchers. As it is virtually impossible to obtain a representative sample of older migrants in the UK (Boneham et al. 1997), a non-probability, snowball sample was constructed (Burholt and Shah 2001). Firstly, the UK sample was drawn from Birmingham in the West Midlands because there are high concentrations of South Asians in particular locations: Punjabi participants were selected from Handsworth and Lozells, Sylheti participants were drawn from Aston and Alum Rock, and Gujarati respondents were selected from Sparkhill and Highgate. Secondly, access to participants was sought via local ethnic associations in the selected areas, that is through temples, mosques, gurdwaras, day centres, various women's groups and other informal meeting places for elders, such as drop-in centres. Thirdly, to supplement lists of participants provided by the community associations, participants were asked to provide names and contact details of other potential study participants.

In South Asia, the samples were drawn from villages in Gujarat and Punjab in India and Sylhet in Bangladesh identified by our Asian colleagues as primary sources of migration to the UK. Within Gujarat, our respondents came from the Kheda district, in Punjab, the study communities were from Jalandhar district and in Sylhet the study community was located in Sylhet Sadar District. In India, a household census was taken in the selected areas from which a stratified (50\% male and $50 \%$ female) random population sample was drawn from all households containing an older person. In Bangladesh, the stratified sample was randomly selected from households from which at least one family member had migrated overseas.

The interview schedule was written in English and based on a pilot project conducted in Bangladesh and with Bangladeshis living in Tower Hamlets, 
London (Burholt et al. 200o). The schedule was translated into Punjabi, Gujarati and Sylheti (using front-back translation methods; Koller et al. 2007). Interviews were conducted between January 2001 and May 2002, in the respondent's native language (Gujarati, Hindi, Punjabi, Urdu or Bangla) and wherever possible in the respondent's own home.

\section{Study variables}

The original 'Families and Migration: Older People from South Asia' project was co-ordinated by Professor G. Clare Wenger, and data were collected to establish network type according to the Wenger Support Network Typology. The Wenger Support Network Typology identifies five types of support networks established through the responses to eight questions on the availability of local kin, frequency of face-to-face interaction with family, friends and neighbours, and community integration (Wenger 1991).

In 'Local Family-dependent Networks', the older person relies on relatives living in the same community. Community involvement is low and networks tend to be small. An older person with this type of network is often widowed and in less good health than those with other types of network.

'Locally Integrated Networks' are associated with helping relationships with local family, friends and neighbours. Older people are usually actively involved in religious and/or community groups and average network size is larger than other network types.

'Local Self-contained Networks' reflect a more privatised householdcentred lifestyle with reliance on neighbours if essential. Older people with this type of network may be childless and in contact with a relative in a different community (e.g. niece, nephew). Community involvement is rare or low key and the network is smaller than most other network types.

'Wider Community-focused Networks' are associated with an absence of local kin but a focus on friends and involvement in community groups. An older person with this type of network is likely to have a long-distance relationship with kin. Engagement in community groups and voluntary organisations means that networks are large.

'Private Restricted Networks' are associated with an absence of local kin and low levels of contact with neighbours and the community. This network type subsumes two types of older person: those who married and have lifestyles that are unconnected with their communities and older people who have withdrawn or become isolated from local involvement (e.g. in the face of poor health). These networks are small.

Isolation was assessed by the reported number of hours spent home alone during the day. This variable was coded as $<3$ hours $(1), \geqslant 3$ and $<6$ hours $(2), \geqslant 6$ and $<9$ hours (3) and $\geqslant 9$ hours (4) (mean=1.78, standard 
deviation $(\mathrm{SD})=1.1)$. The item was collapsed into a dichotomous response ( $\mathrm{o}=$ 'rarely isolated' (up to three hours home alone each day) and 1 = 'isolated for most of the day' (three or more hours alone each day)). Loneliness was assessed using a single-item scale: participants indicated whether they were lonely: never (1), rarely (2), sometimes (3), often (4) or most of the time $(5)($ mean $=2.09, \mathrm{SD}=1.18)$. The scale was reduced to a dichotomous response ( $\mathrm{O}=$ 'rarely or never felt lonely' and $1=$ 'felt lonely sometimes or more often').

\section{Analysis}

We took a structural approach to deriving a new typology that would capture a range of network types relevant to the population under study. We reviewed variables concerned with co-residency, provision and receipt of support (i.e. functional support with: cooking, laundry, household chores, shopping for food, bringing prepared food, transportation to general practitioner, borrowing small items, informal care provision and care when ill; emotional support: in confidant relationships, when unhappy and with personal problems; informational support: with financial matters), and the availability of friends and neighbours in order to select the most appropriate network generator variables that would provide the greatest breadth of network membership (including providers of support, and the landscape of potential care-givers) whilst keeping the number of questions to be asked of participants in future research to a minimum (parsimonious). In summary, we selected nine support network-generating questions (restricted to the identification of network members aged 16 years or more). The questions were (a) Who lives in this household with you? (household membership); (b) How often do you have a chat or do something with one of your friends? After this question the interviewer elicited information on up to five named friends. (c) If you were ill and could not leave the house, is there someone who would look after you? (d) Does anyone go to buy food for you? (e) Does anyone cook for you? (f) Does anyone help you with any other [than laundry or cooking] household chores? (g) If you needed advice about money, is there someone you would ask? (h) If you were feeling unhappy and just wanted someone to talk to, is there someone you would go to? (i) If you were worried about a personal problem, is there someone you would talk to? Older people in this sample were both providers and recipients of help; however, the use of additional questions regarding the provision of help across the 13 areas listed above did not generate additional network members. Every person named in response to the nine 'network generator' questions was subsequently included in the participant's support network. The proportion of the network classified by gender; age (under 
$45,45^{-64}, 65^{+}$); kin and non-kin; formal help; and proximity (living in the participant's household or not) was established. These variables were used in K-means cluster analysis.

In the cluster analysis we ran separate models for two to six clusters. Clusters were classified by iteratively updating cluster centres. The most appropriate cluster model was selected based on a good distribution across cluster types, where the differences in the characteristics of each cluster could be accounted for on a theoretical basis and were comparable with results obtained in other research on network types (e.g. Litwin and Landau 2000; Litwin and Shiovitz-Ezra 2010; Melkas and Jylhä 1996; Stone and Rosenthal 1996).

After deriving network types we examined the main characteristics of each network in terms of the network size and constituent membership, alongside the age, gender, marital status, household size and composition, receipt and provision of help (with regard to all 13 functional and emotional support tasks listed above), community integration and parental status of the network reference person (participant) to arrive at descriptions of each network type.

Preliminary validation of the cluster solution was assessed by examining the association between the new typology and the Wenger Support Network Typology, and difference in distribution of network types between migrants (i.e. those participants living in the $\mathrm{UK}$ ) versus non-migrants (those participants living in South Asia). We compared categorical data using Pearson chi square tests $\left(\chi^{2}\right)$. The difference in means of continuous variables (network criterion, age, receipt and provision of help) among the support network types were compared using one-way analysis of variance (ANOVA).

Two logistic regression models assessed the contribution of support network type to the dependent variables loneliness and isolation, while controlling for other demographic characteristics. Odds ratios showed the relative likelihood that participants with particular characteristics were lonely or isolated.

\section{Results}

Of the five cluster models (comprising two to six clusters), we regarded the four-cluster model as the optimal solution. We examined cluster memberships at different stages of the analysis and selected the four-cluster solution to best represent the data, because the cluster profiles were most interpretable, and they seemed to maximise the differences between and minimise the differences within each cluster. The two-, three-, five- and sixcluster solutions resulted in weaker distributions of cases per cluster and 
ambiguous interpretations of network type characteristics. The defining characteristics of the network types (i.e. the characteristics of network members derived from the variables used in the cluster analysis) are shown in Table 1 and the characteristics of the reference person according to their network type are displayed in Table 2. These data were used to generate the descriptions of each network type that are outlined below.

\section{'Multigenerational Households: Older Integrated Networks'}

Twenty-eight per cent of the sample was assigned 'Multigenerational Households: Older Integrated Networks'. Around three-quarters of older South Asians with 'Multigenerational Households: Older Integrated Networks' were married and around one-quarter were widowed. More than threequarters of older South Asians with these networks lived in multigenerational households, whilst around one-fifth lived with their spouse only. On average, households contained approximately six people. This network was comprised around two-thirds kin to one-third non-kin. On average, older people with these networks received help with eight tasks and provided help with approximately five tasks. Older people with these types of networks were well integrated into the local community: in addition to the relatively high number of friends, over two-thirds attended social or community groups at least occasionally (see Table 2). Although the network included both younger and older members, on average nearly one-half were over $6_{5}$ years old. Therefore, the proportion of the network that were over $6_{5}$ and the level of community involvement of the older person served to differentiate it from the other type of multigenerational household ( see below).

\section{'Multigenerational Households: Younger Family Networks'}

Older people with 'Multigenerational Households: Younger Family Networks' had a household-focused lifestyle and accounted for 27 per cent of the sample. Approximately half of the participants with 'Multigenerational Households: Younger Family Networks' were married and half were widowed. A vast majority of participants with these networks lived in multigenerational households. Thus, these networks had the greatest proportion of network members living in the same household. They had the largest households, containing on average seven people, and the greatest number of living children (on average four). On average, older people with these networks received help with approximately nine tasks and provided help with five tasks. Of all the network types this cluster was the most familyfocused: older people with these networks were least likely to participate in social groups or community meetings, and networks were comprised overwhelmingly of kin members with only around one-tenth of non-kin network 
T A B L E 1. Defining characteristics of network members in the four-cluster model of network types

\begin{tabular}{|c|c|c|c|c|c|c|c|c|}
\hline \multirow[b]{3}{*}{ Network type } & \multirow{3}{*}{$\begin{array}{l}\text { Mean } \\
\text { network } \\
\text { size }\end{array}$} & \multicolumn{7}{|c|}{ Criterion variables ${ }^{1}$} \\
\hline & & \multirow[b]{2}{*}{ Male } & \multicolumn{3}{|c|}{ Age } & \multirow[b]{2}{*}{ Kin } & \multirow{2}{*}{$\begin{array}{l}\text { Formal } \\
\text { services }\end{array}$} & \multirow{2}{*}{$\begin{array}{c}\text { Living } \\
\text { in same } \\
\text { household }\end{array}$} \\
\hline & & & $<45$ years & $45^{-64}$ years & $>65$ years & & & \\
\hline Multigenerational Household: Older Integrated Network & 5.82 & $5^{1.93}$ & $39 \cdot 97$ & 12.84 & $47 \cdot 59$ & $69 \cdot 39$ & 1.42 & $59 \cdot 64$ \\
\hline Multigenerational Household: Younger Family Network & $5 \cdot 57$ & 47.79 & 70.54 & 20.69 & 8.83 & $87 \cdot 79$ & 0.59 & 81.84 \\
\hline Family and Friends Integrated Network & 5.85 & $5^{8.70}$ & 25.89 & $5^{8.80}$ & $15 \cdot 31$ & 48.72 & I.IO & 37.67 \\
\hline Restricted Non-kin Network & $4.8 I$ & 38.57 & $I I .8 I$ & 9.83 & 69.95 & 26.44 & $3 \cdot 75$ & 13.54 \\
\hline All & $5 \cdot 5^{8}$ & 50.20 & 36.27 & 26.73 & 32.49 & 61.03 & 1.50 & $5^{1.4^{2}}$ \\
\hline
\end{tabular}

Notes: 1 . Values are the mean proportion of the network with each characteristic. Analysis of variance: network size $(F=4 \cdot 36, p<0.00) ;$ male $(F=16.05$, $p<0.00) ;<45$ years $(F=287.06, p<0.00) ; 45^{-64}$ years $(F=274.94, p<0.00) ;>65$ years $(F=320.89, p<0.00)$; kin $(F=298.89, p<0.00)$; formal services $(F=4.81, p<0.00)$; living in household $(F=335.21, p<0.00)$. Post-hoc group comparisons - Tukey HSD test: numbers that appear in bold $(e . g .5 .82)$ constitute subsets with the highest values; numbers that appear in italics (e.g. 4.8I) constitute subsets with the lowest values. 
T A B L E 2. Demographic characteristics of participants by support network type: frequencies and cross-tabulations

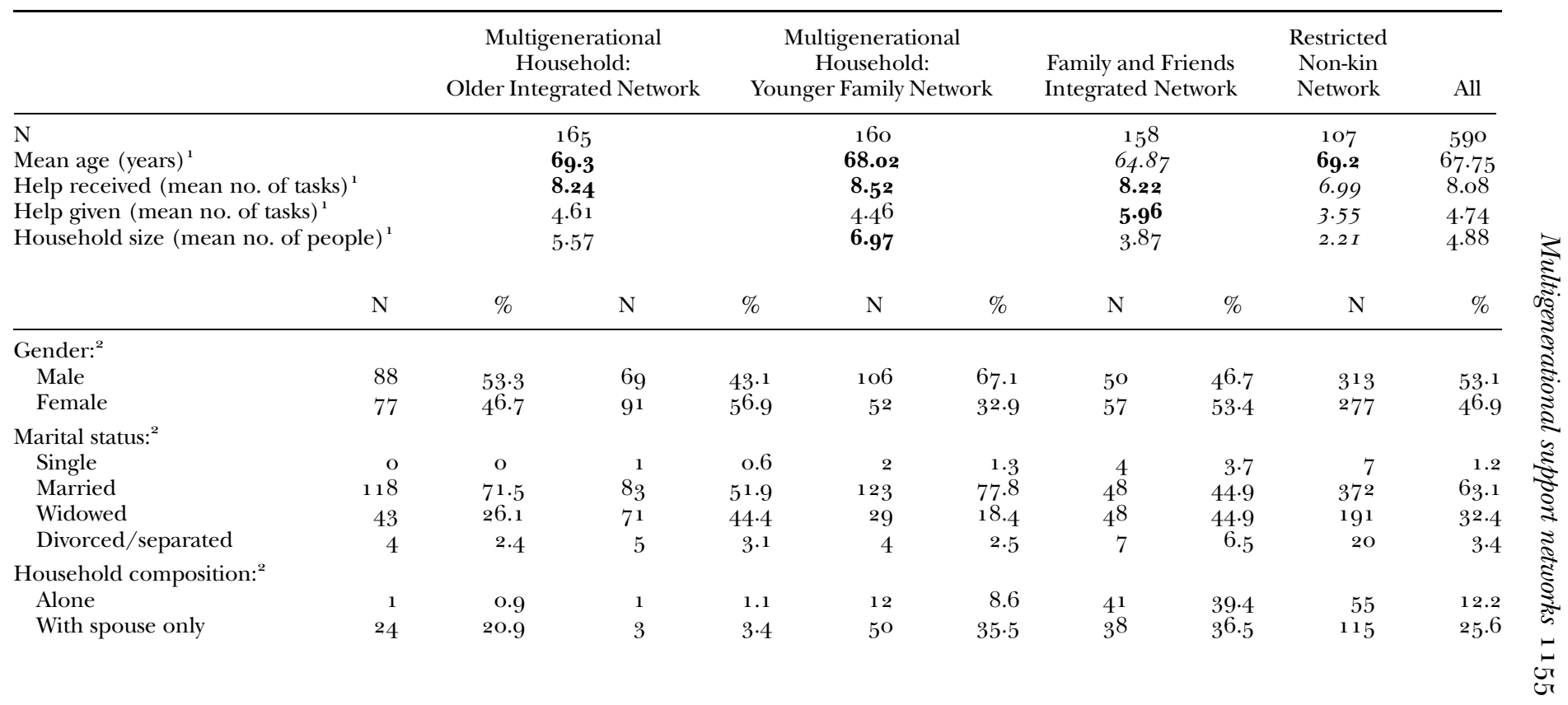


TABLE 2. (Cont.)

\begin{tabular}{|c|c|c|c|c|c|c|c|c|c|c|}
\hline & $\mathrm{N}$ & $\%$ & $\mathrm{~N}$ & $\%$ & $\mathrm{~N}$ & $\%$ & $\mathrm{~N}$ & $\%$ & $\mathrm{~N}$ & $\%$ \\
\hline With other generations & 90 & 78.3 & 85 & $95 \cdot 5$ & 79 & $5^{6.0}$ & 25 & 24.0 & 279 & 62.1 \\
\hline \multicolumn{11}{|l|}{ Childless: $^{2}$} \\
\hline Yes & 4 & 2.4 & 3 & 1.9 & 10 & 6.3 & 13 & 12.1 & 30 & $5 \cdot 1$ \\
\hline No & 161 & 97.6 & 157 & $9^{8.1}$ & $14^{8}$ & $93 \cdot 7$ & 94 & 87.9 & 572 & $94 \cdot 9$ \\
\hline \multicolumn{11}{|l|}{ Community participation: ${ }^{2}$} \\
\hline Never & 49 & 30.4 & 67 & 43.8 & 44 & 28.4 & 27 & 25.7 & 187 & 32.6 \\
\hline At least occasionally & 112 & 69.6 & 86 & $5^{6.3}$ & 111 & 71.6 & 78 & $74 \cdot 3$ & 387 & $67 \cdot 4$ \\
\hline \multicolumn{11}{|l|}{ Religious participation: $^{2}$} \\
\hline Never & 29 & 17.8 & 27 & $17 \cdot 4$ & 9 & $5 \cdot 9$ & 14 & 14.1 & 79 & 13.9 \\
\hline At least occasionally & 134 & 82.2 & 128 & 82.6 & 143 & $94 \cdot 1$ & 85 & 85.9 & 490 & 86.1 \\
\hline
\end{tabular}

Notes: 1. Analysis of variance: age $(F=9.35, p<0.00)$; help received $(F=7.56, p<0.00)$; help given $(F=14.92, p<0.00)$; household size $(F=83.4, p<0.00)$. Post-hoc group comparisons - Tukey HSD test: numbers that appear in bold (e.g. 69.3) constitute subsets with the highest values; numbers that appear italic (e.g. 64.87) constitute subsets with the lowest values.

2. Pearson chi-square: gender $\left(\chi^{2}=20.55\right.$, degrees of freedom $\left.(\mathrm{df})=3, p<0.00\right)$; marital status $\left(\chi^{2}=56.27, \mathrm{df}=9\right.$, $\left.p<0.00\right)$; household composition $\left(\chi^{2}=159.74, \mathrm{df}=6, p<0.00\right)$; childless $\left(\chi^{2}=17.41, \mathrm{df}=3, p<0.00\right)$; community participation $\left(\chi^{2}=15.59, \mathrm{df}=3, p<0.00\right)$; religious participation $\left(\chi^{2}=11.79\right.$; $\mathrm{df}=3, p<0.00)$; migrant status $\left(\chi^{2}=23.66, \mathrm{df}=3, p<0.00\right)$. 
members. This network had the smallest proportion of members over 65 years: overall, a vast majority of network members were under 45 years.

\section{'Family and Friends Integrated Networks'}

Over one-quarter $(26.8 \%)$ of participants were classified as having 'Family and Friends Integrated Networks'. The household size of people with these networks was fairly small (average four persons). More than threequarters of people with 'Family and Friends Integrated Networks' were married, more than one-third lived with a spouse only, while more than onehalf lived in a multigenerational household. Given that households were fairly small, nearly two-thirds of network members lived in a different household. The key difference between this network type and the others was the proportion of non-kin members in the network: networks were comprised nearly equally of family and friends. Furthermore, this network was characterised by high levels of support provided to others. While older people with these networks tended to receive a similar amount as help as those living in either of the multigenerational household types (on average received support for eight tasks), they provided help with six tasks to other people. Network members tended to be aged between 45 and 64 years. Compared to other networks, older people with this type of network were younger (average age 64.7 years) and had a community-facing lifestyle as indicated by the large proportion of friends, and the high proportion that participated in religious organisations or meetings $(94.1 \%)$ at least occasionally.

\section{'Restricted Non-kin Networks'}

Fewer than one-fifth $(18.1 \%)$ of the sample were assigned to 'Restricted Non-kin Networks'. Older people with 'Restricted Non-kin Networks' were nearly equally divided between those that were married and those that were widowed. However, older people that had never married or were divorced and/or separated tended also to be classified as having this type of network. Furthermore, more than one-tenth of older people with restricted non-kin networks were childless. Participants were most likely to either live alone or live with a spouse only (i.e. not in a multigenerational household). Thus, households were small (average two persons) and networks were smaller than others containing on average five members. These small networks had the greatest proportion of non-kin members and the greatest proportion of members that were over $6_{5}$ years old. Older people with these networks received and provided help with the fewest tasks when compared with the other networks. Furthermore, these networks contained the greatest 


\section{$115^{8}$ Vanessa Burholt and Christine Dobbs}

proportion of formal helpers (e.g. paid carers, domestic help) although this was a fairly low proportion of the network as a whole $(4 \%)$.

\section{Differences between clusters, migrant status and the Wenger Support Network Typology}

Table 3 displays the results of the first step of preliminary validation, and the cross-tabulation of the four-cluster model of network types with migrant status and the Wenger Support Network Typology. Firstly, there are significant differences between the distribution of network types for migrants and non-migrants. This is manifest in the smaller proportion of migrants with 'Multigenerational Households: Younger Family Networks' and a greater proportion with 'Restricted Non-kin Networks' when compared to their counterparts in the country of origin. Secondly, whilst the new clusters (network types) have some association with the Wenger network types there are also some crucial differences.

'Multigenerational Households: Younger Family Networks' were similar to the family-dependent network identified by Wenger as over two-thirds $(69.3 \%)$ of this cluster was identified as family dependent. However, we have also identified a 'sub-type' of family-dependent network that was evident for South Asian elders and this was 'Multigenerational Households: Older Integrated Networks'. More than half $(55.1 \%)$ of participants with this network type were also classified as family dependent. The key differences between the two networks with regard to the degree of community participation are not captured in the Wenger typology where the classification is predominantly influenced by the proximity of family members to the participant.

'Family and Friends Integrated Networks' were associated with three of the Wenger network types (subsuming all of those with high levels of community/non-kin involvement). It comprised nearly one-third of all locally integrated networks $(32.4 \%)$, over one-half of local self-contained networks $(58.8 \%)$ and more than two-fifths $(43.5 \%)$ of wider communityfocused networks. As noted above, the 'Family and Friends Integrated Networks' were characterised by high levels of receipt and provision of support to others, suggesting that older people with these networks are well connected to others (especially non-kin) in the community. This is contrary to the description of the local self-contained network which is characterised as private and household focused.

'Restricted Non-kin Networks' map on to Wenger's private restricted networks. The cross-tabulation showed that $45.8 \%$ of all private restricted networks fell in this group. However, it is important to note that $72.9 \%$ of the sample with 'Restricted Non-kin Networks' were classified as 
T A B L E 3. Four-cluster support network typology cross-tabulated with migrant status and Wenger Support Network Typology

\begin{tabular}{|c|c|c|c|c|c|c|c|c|c|c|}
\hline & \multicolumn{2}{|c|}{$\begin{array}{l}\text { Multigenerational } \\
\text { Household: Older } \\
\text { Integrated Network }\end{array}$} & \multicolumn{2}{|c|}{$\begin{array}{l}\text { Multigenerational } \\
\text { Household: } \\
\text { Younger Family } \\
\text { Network }\end{array}$} & \multicolumn{2}{|c|}{$\begin{array}{l}\text { Family and } \\
\text { Friends } \\
\text { Integrated } \\
\text { Network }\end{array}$} & \multicolumn{2}{|c|}{$\begin{array}{l}\text { Restricted } \\
\text { Non-kin } \\
\text { Network }\end{array}$} & \multicolumn{2}{|c|}{ All } \\
\hline & $\mathrm{N}$ & $\%$ & $\mathrm{~N}$ & $\%$ & $\mathrm{~N}$ & $\%$ & $\mathrm{~N}$ & $\%$ & $\mathrm{~N}$ & $\%$ \\
\hline \multicolumn{11}{|l|}{ Migrant status: ${ }^{1}$} \\
\hline No & 85 & $5^{1.5}$ & 94 & $5^{8.8}$ & 77 & 48.7 & 31 & 29.0 & 287 & 48.6 \\
\hline Yes & 80 & 48.5 & 66 & $4^{1.2}$ & 81 & $5^{1 \cdot 3}$ & 76 & 71.0 & 303 & $5^{1.4}$ \\
\hline Total & 165 & & 160 & & $15^{8}$ & & 107 & & 590 & \\
\hline \multicolumn{11}{|c|}{ Wenger Support Network Typology: ${ }^{1}$} \\
\hline Family dependent & 87 & 55.1 & 104 & $69 \cdot 3$ & 53 & $34 \cdot 0$ & 29 & 28.2 & 273 & 48.1 \\
\hline Locally integrated & 53 & $33 \cdot 5$ & $4^{1}$ & $27 \cdot 3$ & 76 & $42 \cdot 9$ & 46 & $44 \cdot 7$ & 207 & $3^{6.5}$ \\
\hline Local self-contained & 3 & 1.9 & 1 & 0.7 & 10 & 6.4 & 3 & 2.9 & 17 & 3.0 \\
\hline Wider community focused & 9 & $5 \cdot 7$ & 20 & 12.8 & 3 & 2.0 & 14 & 13.6 & 46 & 8.1 \\
\hline Private restricted & 6 & 3.8 & 1 & 0.7 & 6 & 3.8 & 11 & 10.7 & 24 & 4.2 \\
\hline Total & $15^{8}$ & & $15^{\circ}$ & & $15^{6}$ & & 103 & & $5^{67^{2}}$ & \\
\hline
\end{tabular}

Notes: 1. Pearson chi-square: migrant status $\left(\chi^{2}=23.66\right.$, degrees of freedom $(\mathrm{df})=3, p<0$. oo $)$; Wenger Support Network Typology $\left(\chi^{2}=78.38\right.$, df $=12$, $p<0.00)$, however five cells $(25 \%)$ had a value less than 5 indicating that results of the test were not particularly robust.

2. Excludes 23 participants who were classified 'inconclusive' using the Wenger Support Network Typology. 
T А в L Е 4. Support network type, background characteristics and wellbeing (loneliness and isolation) among older South Asians aged 55+ years: logistic regressions

\begin{tabular}{|c|c|c|c|c|}
\hline \multirow[b]{2}{*}{ Categories $^{1}$} & \multicolumn{2}{|c|}{ Lonely $^{2}$} & \multicolumn{2}{|c|}{ Isolated $^{2}$} \\
\hline & OR & $95 \% \mathrm{CI}$ & OR & $95 \% \mathrm{CI}$ \\
\hline $\mathrm{N}$ & \multicolumn{2}{|l|}{$5^{84}$} & \multicolumn{2}{|c|}{578} \\
\hline \multicolumn{5}{|l|}{ Support network: } \\
\hline $\begin{array}{l}\text { Multigenerational Household: } \\
\text { Older Integrated Network }\end{array}$ & 0.61 & $0.36,1.02$ & $0.34^{* *}$ & $0.16,0.72$ \\
\hline $\begin{array}{l}\text { Multigenerational Household: } \\
\text { Younger Family Network }\end{array}$ & 0.61 & $0.36,1.05$ & 0.70 & $0.35,1.40$ \\
\hline Family and Friends Integrated Network & $0.42^{* * *}$ & $0.25,0.71$ & $0.43^{*}$ & $0.22,0.85$ \\
\hline Age: $55^{-69}$ & 0.77 & $0.53,1.11$ & $0.59^{*}$ & $0.35,0.99$ \\
\hline Gender: Male & 1.02 & $0.68,1.5^{1}$ & 1.63 & $0.92,2.90$ \\
\hline \multicolumn{5}{|l|}{ Marital status: } \\
\hline Never married & 1.79 & $0.23,10.79$ & 6.66 & $0.79,5^{6.57}$ \\
\hline Married & 0.75 & $0.28,1.99$ & 0.67 & $0.14,3.15$ \\
\hline Widowed & 1.64 & $0.61,4.43$ & $3 \cdot 34$ & $0.7^{2}, 15 \cdot 5^{1}$ \\
\hline
\end{tabular}

Notes: 1. Reference categories: Support network: restricted non-kin network; Age: 70+; Gender: female; Marital status: divorced/separated; Loneliness (o); Isolated (o).

2. Outcome variables: Loneliness: $\mathrm{O}=$ 'rarely or never felt lonely', $1=$ 'felt lonely sometimes or more often'; Isolation: $\mathrm{o}=$ 'rarely isolated', 1 = 'isolated for most of the day'. OR: odds ratio. CI: confidence interval.

Significance levels: $* p<0.05, * * p<0.01, * * * p \leqslant 0.001$.

having either locally integrated or family-dependent networks according to the Wenger Support Network Typology, the latter being more robust rather than vulnerable networks. The new typology classified nearly a fifth $(18.2 \%)$ of the study population as members of 'Restricted Non-kin Networks' compared to only 4 per cent of the sample classified in the most vulnerable network using the Wenger Support Network Typology.

\section{Predicting wellbeing outcomes: isolation and loneliness}

Table 4 displays the results of the second step of preliminary validation. This looks at the ability of the clusters (network types) to predict loneliness and isolation. With regard to demographic characteristics, only age predicted isolation (but not loneliness). After controlling for the demographic characteristics (age, gender and marital status), support networks maintained an independent association with both outcome variables (loneliness and isolation) in logistic regression models. When compared to the reference category ('Restricted Non-kin Networks') those with 'Family and Friends Integrated Networks' had significantly lower odds of being lonely or isolated; and those with 'Multigenerational Households: Older Integrated Networks' 
were less likely to report isolation. The analysis indicated 'Restricted Non-kin Networks' were most vulnerable in terms of loneliness and isolation.

\section{Discussion}

Using a structural approach to the development of a support network typology, this analysis has identified four support networks among older South Asians. Based on the characteristics of the network members, and the reference person, the support networks were named 'Multigenerational Households: Older Integrated Networks', 'Multigenerational Households: Younger Family Networks', 'Family and Friends Integrated Networks' and 'Restricted Non-kin Networks'. The network types are differentiated on the structure of the networks, community integration, and the quantity of support provided and received. In addition to the structural analysis, preliminary validation of the cluster solution suggests that the new typology differentiates between migrants and non-migrants, and detects variation between networks that have intergenerational co-residence in common.

The new typology distinguishes between two types of support networks primarily associated with multigenerational households. While older people with 'Older Integrated Networks' have community-facing lifestyles, those with 'Younger Family Networks' are home-focused, comprising younger people and have less community interaction. More than half of the participants with 'Family and Friends Integrated Networks' also live in multigenerational households, and these networks differ from the others in the proportion of non-kin members and the degree of support that the older reference person provides to others. Litwin (2001) found that older people with diverse and friends-based networks had the highest morale, while those with restricted and family networks had the lowest morale. Thus, it is important that the network types described in this paper are distinct in terms of their community integration and mix of kin and non-kin. Future research using the network typology could explore whether having a variety of people in one's network is better for psychological wellbeing.

The new network typology performs better than the Wenger Support Network Typology in identifying vulnerable or fragile networks. Were we to have relied on the Wenger Support Network Typology we would have concluded that only a small minority of South Asian elders (4.2\%) were embedded in private restricted-support networks - the least robust network type, and the one most likely to require formal support services. In comparison, the new typology classified nearly a fifth $(18.2 \%)$ of the study population as members of 'Restricted Non-kin Networks', the most vulnerable network in the new cluster typology. Whilst the four-cluster 


\section{Vanessa Burholt and Christine Dobbs}

model has some important similarities with the Wenger Support Network Typology, by developing a new network typology with a population with a preponderance of multigenerational households we have arrived at a different constellation of network types.

The contrast in the distribution of network types between migrants and non-migrants also lends support to the validity of the four-cluster model. Weinreich (2009) has suggested that some migrants retain aspects of their culture of heritage whilst also acquiring a number of elements from the culture in the country of residence (enculturation). Similar proportions of 'Multigenerational Households: Older Integrated Networks' and 'Family and Friends Integrated Networks' in the UK and South Asia suggest that ethnic heritage has influenced community participation and the informal support of older migration in the UK. However, the smaller proportion of migrants with 'Multigenerational Households: Younger Family Networks' and the greater proportion with 'Restricted Non-kin Networks' compared to older people living in South Asia suggest that older migrants (or the secondand third-generation members of their families) may have adopted mainstream cultural norms with regard to smaller and/or nuclear households, independence and autonomy, rather than a preference for extended households and reliance on familial support. Alternatively, pragmatic reasons may lie behind the differences in network types. Older people with 'Restricted Non-kin Networks' have the greatest proportion of childless participants, suggesting that migration may have disrupted childbearing and impacted on family formation ( see also Burholt 2004 $a$ for gendered patterns of migration and family reunification). Secondly, migrants may have particular ambitions for their children's education and subsequent employment. The ability to relinquish expectations for care from adult children provides the extended family with more human resources that may facilitate the potential for social and economic mobility of future generations (Burholt and Dobbs 2010).

While meaningful differences between the new typology, the Wenger Support Network Typology, migrants and non-migrants provide support for the preliminary validity of the four-cluster model, the derived network typology also has additional predictive power. The logistic regression models indicated that 'Restricted Non-kin Networks' were particularly vulnerable. Older South Asians with these networks were more likely to be lonely and isolated compared to those embedded in the other types of support networks. The results of this research have important implications for forecasting formal services provision based on the distribution of support network types.

In the UK indigenous or majority population, network type has been found to be related to the use of statutory domiciliary services and related to community type (Wenger 1991; Wenger and St Leger 1992). Thus, the 
distribution of network types has been assumed to have significant implications for the allocation and dispersal of care in the community. When traditional support network typologies are used with older people from familistic cultures, the distributions may be skewed towards more robust network types. The amplification of the proportion of older people with robust networks may contribute to tenacious stereotyping - that they prefer to 'look after their own' - and may reinforce institutional racism: the belief of service providers that there is little that needs to be done in the way of service provision (MacPherson 1999; Willis 2010). Service planning built on this evidence could underestimate the support needs of some older people who may be isolated and lonely and with limited informal sources of help. The new network typology identifies a greater proportion of older people with vulnerable networks, and could positively contribute to service planning for migrant communities and in countries where co-residency is common.

In addition to the consequences for policy and practice, the development of this network typology has implications for future research and theory generation. As noted in the introduction, there is little empirical evidence to refute or support the tenets of modernisation theory or minority group theory in relation to supposed impacts on support networks. The measurement instrument developed in this paper could be used to test hypotheses concerning both theories. In relation to minority group theory, the typology could be used to establish whether different patterns of informal support are related to social exclusion, health and social inequalities for older migrants within familistic cultures. It would also be desirable to carry out longitudinal studies to understand the dynamics of support networks in countries with familistic values with particular reference to testing modernisation theory. For example, it would be useful to track changes in the prevalence of each type of network to see if these correspond to social transformations (such as urbanisation and increased labour force participation of women). Similarly, it would be interesting to undertake systematic longitudinal analysis of the support networks of migrant groups to examine the extent to which family forms 'modernise' through acculturation (Redfield, Linton and Herskovitz 1936) or enculturation (Weinreich 2009). In respect of both minority theory and modernisation theory, longitudinal analysis may help to move beyond these rather dated gerontological debates where older people within familistic cultures are generally considered to be passive recipients of care, and subject to social forces beyond their control (such as ageism, modernisation) to more current critical theoretical perspectives on ageing, that take into account the lifecourse, access to resources and the context in which these are experienced.

There are some limitations to this analysis that relate to the cultural context of the sample and the sampling methods, the single-item outcome 


\section{Vanessa Burholt and Christine Dobbs}

variables used in the analysis, and the cultural specificity of the typology. Firstly, the support network typology has been developed with South Asian elders living in the UK and South Asia. While we believe that this typology would be useful applied to other older populations who have a high prevalence of multigenerational households, we cannot rule out that the observed associations between network types and wellbeing factors, and the distribution of network types are not consistent across other cultures (e.g. Litwin 2009). The snowball sampling method used to draw the UK South Asian sample may have resulted in an under-representation of 'Restricted Non-kin Networks' by omitting more isolated individuals from the sample. Furthermore, the setting (Birmingham, UK) has high concentrations of particular ethnic groups that may have influenced the distribution of network types. Living in an area with a single predominant ethnic group (sometimes referred to as an 'ethnic enclave') promotes solidarity through large, strong social networks and is likely to impact on social integration into mainstream society by decreasing the returns from conformity to expected behaviour and norms (Salant and Lauderdale 2001). The networks of migrants from familistic cultures living in other areas of the UK may be either more traditional and inward-facing, for example rejecting mainstream culture and emphasising ethnic heritage, or more individualistically oriented having broken away from 'traditional' norms in ethnically concentrated areas (Harries, Richardson and Soteri-Proctor 2008). Currently, the fourcluster network typology is being assessed with older people from six migrant groups in England and Wales, but we also recommend that the network typology is tested elsewhere in the world with familistic indigenous and migrant populations. Secondly, our study was limited because the validity of the typology has been assessed (partly) by its ability to predict the single-item dependent variables loneliness and isolation. Future studies could investigate further whether network types predict composite measures of loneliness and isolation. Thirdly, it is beyond the scope of this paper to explore the cultural specificity of the network typology between or within groups of South Asians (Gujaratis, Punjabis and Sylhetis). We are keen to note that we do not advocate reporting generalisations based on data amalgamation of ethnicities, cultures and personal histories, and a paper on the distribution of network types for each South Asian group (Gujaratis, Punjabis and Sylhetis) is forthcoming. Despite the aforementioned limitations, the new cluster model provides a useful tool for the consideration of support network types of older people and has potential for use elsewhere in communities and cultures with a preponderance of multigenerational households. The support network typology focuses on the membership of the support network, and because the sample includes a large proportion of participants with multigenerational households, unlike the existing 
typologies developed with samples where this living arrangement is rare, it is able to deal with nuanced differences between multigenerational households and identify a greater proportion of vulnerable networks.

\section{Acknowledgements}

We would like to acknowledge funding from the Department for International Development, UK (Project: ESA3 15 ) for the original data collection in the UK and South Asia. Our thanks is also extended to the original project co-ordinator, Professor G. Clare Wenger, and the collaborators and the institutions that they were based in at the time of the research: Abdul Awwal Biswas, Shahjahal University, Sylhet, Bangladesh; Parul Dave and Indira Mallya, Maharajah's University, Vadodara, Gujarat; N. S. Sodhi, Punjabi University, Patiala, Punjab, India; Zahida Shah, Bangor University, UK; and Shubha Soneja, HelpAge India, Delhi. We are also grateful to colleagues at the Irish Centre for Social Gerontology, National University of Ireland Galway, for hosting a visiting fellowship in 2011/12 during which time this paper was completed. This study was granted ethical approval by the Research Committee in the College of Human and Health Science at Swansea University. We gratefully acknowledge funding from the Economic and Social Research Council, UK (grant number: RES-ooo-22-3312) that supported the secondary data analysis presented in this paper. Both authors have made a substantial contribution to the conception, design, analysis and interpretation of data. Furthermore, both authors have contributed to the drafting of the article. There are no conflicts of interest.

\section{References}

Baltes, M. M. and Carstensen, L. L. 2003. The process of successful aging: selection, optimization, and compensation. In Staudinger, U. M. and Lindenberger, U. (eds), Understanding Human Development: Dialogues with Life-span Psychology. Kluwer Academic, Dordrecht, The Netherlands, 81-104.

Beauchemin, C. and González-Ferrer, A. 2011 . Sampling international migrants with origin-based snowballing method: new evidence on biases and limitations. Demographic Research, 25, 3, 103-34.

Blanchflower, D. G. and Oswald, A. J. 2004. Well-being over time in Britain and the USA. Journal of Public Economics, 88, 7-8, 1359-86.

Boneham, M. A., Williams, K. E., Copeland, J. R. M., McKibbin, P., Wilson, K., Scott, A. and Saunders, P.A. 1997. Elderly people from ethnic minorities in Liverpool: mental illness, unmet need and barriers to service use. Health and Social Care in the Community, 5, 3, 173-80.

Burholt, V. 2004a. The settlement patterns and residential histories of older Gujaratis, Punjabis and Sylhetis in Birmingham, England. Ageing $\mathcal{E} \odot$ Society, 24, 3, $383-410$.

Burholt, V. 2004 $b$. Transnationalism, economic transfers and families' ties: intercontinental contacts of older Gujaratis, Punjabis and Sylhetis in Birmingham with families abroad. Ethnic and Racial Studies, 27, 5, 800-29.

Burholt, V. and Dobbs, C. 2010. Care-giving and care-receiving relationships of older South Asians: functional exchange and emotional closeness. GeroPsych: The Journal of Gerontopsychology and Geriatric Psychiatry, 23, 4, $215^{-2} 5$. 


\section{Vanessa Burholt and Christine Dobbs}

Burholt, V. and Shah, Z. 2001. Older people from South Asia: cross-national sample selection in India, Bangladesh and United Kingdom. Generations Review, 1 1, 4, 4-7.

Burholt, V. and Wenger, G. C. 2004. Migration from South Asia to the UK and the maintenance of transnational intergenerational relationships. In Silverstein, M., Giarrusso, R. and Bengtson, V. L. (eds), Intergenerational Relations Across Time and Place. Springer Annual Review of Gerontology and Geriatrics. Springer Publishing, New York, $153-76$.

Burholt, V., Wenger, G. C., Biswas, A. A., Dave, P., Mallya, I., Sohdi, N. S., Shah, Z. and Soneja, S. 2003. Families and Migration: Older People from South Asia. Final Report. Department for International Development, London.

Burholt, V., Wenger, G. C., Scott, A., Yahya, B. and Roy, S. 200o. Bangladeshi immigration to the UK: older people's support networks in the sending and receiving countries. Quality in Ageing, 1, 2, $18-30$.

Cattell, V. 2001. Poor people, poor places, and poor health: the mediating role of social networks and social capital. Social Science and Medicine, 52, 10, 1501-16.

Cheng, S.-T., Lee, C. K. L., Chan, A. C. M., Leung, E. M. F. and Lee, J.-J. 2oog. Social network types and subjective well-being in Chinese older adults. Journal of Gerontology: Psychological Sciences, 64B, 6, 713-22.

Cheng, S., Li, K., Leung, E. M. and Chan, A. C. 2011. Social exchanges and subjective well-being: do sources of positive and negative exchanges matter? Journals of Gerontology: Psychological Sciences and Social Sciences, 66B, 6, 708-718.

Cooper, C., Bebbington, P. and Livingston, G. 2010. Cognitive impairment and happiness in old people in low and middle income countries: results from the 10/66 study. Journal of Affective Disorders, 13o, 1/2, 198-204.

Cowgill, D. O. 1986. Aging Around the World. Wadsworth, Belmont, California.

Diener, R., Diener, M. and Diener, C. 1995. Factors predicting the subjective wellbeing of nations. Journal of Personality and Social Psychology, 69, $5,85^{1-64}$.

Dubova, S. V., Pérez-Cuevas, R., Espinosa-Alarcón, P. and Flores-Hernández, S. 2010. Social network types and functional dependency in older adults in Mexico. BMC Public Health, 1o, 104.

George, L. K. 2010. Still happy after all these years: research frontiers on subjective well-being in later life. Journals of Gerontology: Social Sciences, $\mathbf{6}_{5} \mathbf{B}, 3,33^{1-9}$.

Golden, J., Conroy, R. M. and Lawlor, B. A. 2009. Social support network structure in older people: underlying dimensions and association with psychological and physical health. Psychology, Health $\mathcal{E}^{\circ}$ Medicine, 14, 3, 280-9o.

Harries, B., Richardson, L. and Soteri-Proctor, A. 2008. Housing Aspirations for a New Generation. Perspectives from White and South Asian British Women. Joseph Rowntree Foundation, York, UK.

Hendricks, J. and Hendricks, C. 1977. Aging in Mass Society. Winthrop Publishers, Cambridge, Massachusetts.

Henry, D. B., Tolan, P. H. and Gorman-Smith, D. 2005. Cluster analysis in family psychology research. Journal of Family Psychology, 19, 1, $121-32$.

Holmes, E. R. and Holmes, L. D. 1995. Other Cultures, Elder Years. Sage, London.

Keyes, C. L. M. 1998. Social well-being. Social Psychology Quarterly, 61, 2, $121-40$.

Kite, M. E., Stockdale, G. D., Whitley, B. E. and Johnson, B. T. 2005. Attitudes toward younger and older adults: an updated meta-analytic review. Journal of Social Issues, 61, 2, 241-66.

Koller, M., Aaronson, N. K., Blazeby, J., Bottomley, A., Dewolf, L., Fayers, P., Johnson, C., Ramage, J., Scott, N., West, K., and on behalf of the EORTC Quality of Life Group 2007. Translation procedures for standardised quality of life questionnaires: the European Organisation for Research and Treatment of Cancer (EORTC) approach. European Journal of Cancer, 43, 12, 1810-20. 
Lawton, M. P. 1975. The Philadelphia Geriatric Center Morale Scale: a revision. Journal of Gerontology, 30, 1, 85-9.

Lawton, M. P. 1983. Environment and other determinants of well-being in older people. The Gerontologist, 23, 4, 349-57.

Levin, J. and Levin, W. 1982. Ageing: Prejudice and Discrimination Against the Elderly. Wadsworth, Belmont, California.

Litwin, H. 1995. The social network of elderly immigrants: an analytic typology. Journal of Aging Studies, 9, 2, $155^{-74}$.

Litwin, H. 1997a. Support network type and health services utilization. Research on Aging, 19, 3, 274-99.

Litwin, H. $1997 b$. The network shifts of elderly immigrants: the case of Soviet Jews in Israel. Journal of Cross-cultural Gerontology, 1 2, 1, 45-6o.

Litwin, H. 2001. Social network type and morale in old age. The Gerontologist, 41, 4, $5^{16-24 .}$

Litwin, H. 2009. Social networks and well-being: a comparison of older people in Mediterranean and non-Mediterranean countries. Journals of Gerontology: Social Sciences, $\mathbf{6}_{\mathbf{5}} \mathbf{B}, 5,599-608$.

Litwin, H. and Landau, R. 20oo. Social network type and social support among the old-old. Journal of Aging Studies, 14, 2, 213-28.

Litwin, H. and Shiovitz-Ezra, S. 2010. Social network type and subjective well-being in a national sample of older Americans. The Gerontologist, 51, 3, 379-86.

Liu, W. T. and Kendig, H. 200o. Critical issues of caregiving: East-West dialogue. In Liu, W. T. and Kendig, H. (eds), Who Should Care for the Elderly? An East-West Value Divide. Singapore University Press, Singapore, 1-23.

Lowenstein, A. 2002. Solidarity and conflicts in coresidence of three generational immigrant families from the former Soviet Union. Journal of Aging Studies, 16, 3, $221-41$.

Lubben, J., Blozik, E., Gillmann, G., Iliffe, S., Von Renteln Kruse, W., Beck, J. C. and Stuck, A. E. 2006. Performance of an abbreviated version of the Lubben Social Network Scale among three European community-dwelling older adult populations. The Gerontologist, 46, 4, 503-13.

Lubben, J. E. and Gironda, M. W. 20oo. Social support networks. In Osterweil, D., Brummel-Smith, K. and Beck, J. C. (eds), Comprehensive Geriatric Assessment. McGraw-Hill, New York, $121-37$.

Lubben, J. and Gironda, M. 2003. Centrality of social ties to the health and wellbeing of older adults. In Berkman, B. and Harooytan, L. K. (eds), Social Work and Health Care in an Aging World. Springer, New York, 319-50.

Lubben, J. and Gironda, M. 2004. Measuring social networks and assessing their benefits. In Phillipson, C., Allan, G. and Morgan, D. (eds), Social Networks and Social Exclusion: Sociological and Policy Perspective. Ashgate, Aldershot, UK, 20-34.

MacPherson, W. 1999. The Stephen Lawrence Inquiry: Report of an Inquiry by Sir William Macpherson of Cluny. The Stationery Office, London.

Melkas, T. and Jylhä, M. 1996. Social network characteristics and social network types among elderly people in Finland. In Litwin, H. (ed.), The Social Network of Older People: A Cross-national Analysis. Praeger, Westport, Connecticut, 99-116.

Minkler, M. 1999. Intergenerational households headed by grandparents: contexts, realities and implications. Journal of Aging Studies, 13, 2, 199-2 18.

Ng, A. C. Y., Phillips, D. R. and Lee, W. K.-M. 2002. Persistence and challenges to filial piety and informal support of older persons in a modern Chinese society: a case study in Tuen Mun, Hong Kong. Journal of Aging Studies, 16, 2, $135^{-53}$.

Paice, J. 2002. Living arrangements and ethnicity of Australia's older population. Journal of Population Research, Special Edition September, 159-268. 


\section{Vanessa Burholt and Christine Dobbs}

Paim, L. 1995. Definitions and measurements of wellbeing: a review of literature. Journal of Economic and Social Measurement, 21, 297-309.

Palmore, E. 1978. Are the aged a minority group? Journal of the Amerian Geriatric Society, 26, 5, $214^{-7}$.

Palmore, E. and Manton, T. 1973. Ageism compared to racism and sexism. Journal of Gerontology, 28, 3, 363-9.

Redfield, R., Linton, R. and Herskovitz, M.J. 1936. Memorandum for the study of acculturation. American Anthropologist, 38, 1, 149-52.

Rogler, L. H. 1994. International migrations: a framework for directing research. American Psychologist, 49, 8, 701-8.

Rosenthal, C. 1986. Family supports in later life: does ethnicity make a difference? The Gerontologist, 26, 1, 19-24.

Ruggles, S. and Huggeness, M. 2008. Intergenerational coresidence in developing countries. Population and Development Review, 34, 2, 253-81.

Ryff, C. and Keyes, C. 1995. The structure of psychological well-being revisited. Journal of Personality and Social Psychology, 69, 4, 719-27.

Salant, T. and Lauderdale, D.S. 2001. Measuring culture: a critical review of acculturation and health in Asian immigrant populations. Social Science and Medicine, 57, 1, 71-90.

Schermerhorn, R.A. 1970. Comparative Ethnic Relations: A Framework for Theory and Research. Random House, New York.

Sereny, M. 2011 . Living arrangements of older adults in China: the interplay among preferences, realities, and health. Research on Aging, 33, 2, 172-204.

Shrestha, S. 2006. Social support networks of older people in Newar ethnic community of Nepal. Unpublished Masters thesis, Bangor University, Bangor, UK.

Silverstein, M., Burholt, V., Wenger, G. C. and Bengtson, V. L. 1998. Parent-child relations among very old parents in Wales and the United States: a test of modernization theory. Journal of Aging Studies, 1 2, 4, 387-409.

Slocum-Gori, S. L., Zumbo, B. D., Michalos, A. C. and Diener, E. 20og. A note on the dimensionality of quality of life scales: an illustration with the Satisfaction with Life Scale (SWLS). Social Indicators Research, 92, 3, 489-96.

Stone, L. and Rosenthal, C. 1996. Profiles of the social networks of Canada's elderly: an analysis of 1990 general social survey data. In Litwin, H. (ed.), The Social Networks of Older People: A Cross-national Analysis. Praeger, Westport, Connecticut, 77-97.

Thornton, M. C. and White-Means, S. I. 2000. Race versus ethnic heritage in models of family economic decisions. Journal of Family and Economic Issues, 21, 1, $65^{-86 .}$

Tomassini, C., Glaser, K., Wolf, D. A., Broese van Groenou, M. I. and Grundy, E. 2004. Living arrangements among older people: an overview of trends in Europe and the USA. Population Trends, $115,24-34$.

Treas, J. and Mazumdar, S. 2002. Older people in America's immigrant families: dilemmas of dependence, integration and isolation. Journal of Aging Studies, 16, 3 , $243^{-} 5^{8}$.

Veenhoven, R. 2004. Subjective measures of well-being. Discussion Paper No. 2004/o7, World Institute for Development Economics Research, United Nations University, Helsinki, Finland.

Weinreich, P. 2009. 'Enculturation,' not 'acculturation': conceptualising and assessing identity processes in migrant communities. International Journal of Intercultural Relations, 33, 2, 124-39.

Wenger, G. C. 1991. A network typology, from theory to practice. Journal of Aging Studies, 5, 1, 147-62. 
Wenger, G. C. 1997. Social networks and the prediction of elderly people at risk. Aging and Mental Health, 1, 4, 31 1-20.

Wenger, G. C. and Burholt, V. 2004. Changes in levels of social isolation and loneliness among older people in a rural area: a twenty-year longitudinal study. Canadian Journal on Aging, 23, 2, $115^{-27}$.

Wenger, G. C. and Liu, J.M. 1999. Support networks in Beijing (China) and Liverpool (UK): differences and similarity. Hallym International Journal of Aging, $\mathbf{1}$, 2, 47-57.

Wenger, G. C. and St Leger, F. 1992. Community structure and support network variations. Ageing $\mathcal{E}^{2}$ Society, 1 2, 2, 213-36.

Wenger, G. C. and Tucker, I. 2002. Using network variation in practice: identification of support network type. Heath E Social Care in the Community, 10, 1, 28-35.

Willis, R. 2008. Advantageous inequality or disadvantageous equality? Ethnicity and family support among older people in Britain. Ethnicity and Inequalities in Health and Social Care, 1, 2, 18-23.

Willis, R. 2010. Myths and statistics: an exploration of the assumptions connected to ethnicity and family support. Radical Statistics, 10o, 1 7-27.

Wilmoth, J. 2001. Living arrangements among older immigrants in the United States. The Gerontologist, 41, 2, 228-38.

Wirth, L. 1945. The problem of minority groups. In Linton, R. (ed.), The Science of Man in the World Crisis. Columbia University Press, New York, 347-72.

Yeo, G. and Gallagher-Thompson, D. (eds) 2006. Ethnicity and the Dementias. Second edition, Routledge, New York.

Accepted I 2 December 2012 ; first published online I 2 February 2013

Address for correspondence:

Vanessa Burholt, Centre for Innovative Ageing,

College of Human and Health Sciences, Swansea University,

Singleton Park, Swansea SA2 8PP, UK.

E-mail: v.burholt@swansea.ac.uk 